\title{
A Case of Intravaginal Foreign Body
}

\author{
Brig S Chopra, vsm*, Lt Col Y Singh ${ }^{+}$, Sqn Ldr N Magon* \\ MJAFI 2010; 66 : 266-268
}

Key Words: Foreign body in the vagina; Vaginal discharge; Sexual abuse

\section{Introduction}

$\mathrm{V}$ aginal discharge in women of all age groups may be due to variety of causes. Intravaginal foreign body retained for long duration can be a diagnostic dilemma. This report presents the case of a lady who had an intravaginal foreign body for unknown duration. Recurrent, unremitting, foul-smelling, bloody vaginal discharge in a woman should alert the clinician to the possibility of a retained vaginal foreign body. A case of intravaginal foreign body is reported for the diagnostic dilemma it can present, especially with carcinoma cervix.

\section{Case Report}

A 50 years old menopausal lady reported to the outpatient department (OPD) with complaints of foul smelling blood stained discharge since three months. She had no other associated complaints. She was seen by a practitioner and diagnosed as a case of cancer of cervix. She was menopausal for the last five years and had two normal deliveries.

On taking detailed history, she revealed that she had a marital discord and was living separately from her husband for last 15 years. She also gave history of frequent beating and physical assault by her husband preceding their separation. She denied any history of any legal or illegal abortion. She gave history of taking some treatment for white discharge three months back, records for which were not available.

On examination, her general and abdominal examination did not reveal any significant finding. On per speculum examination, there was intensely putrid and foul smelling blood stained discharge in vagina, typical of carcinoma of cervix. Cervix was pulled up and towards right and a streak of silver was seen in the posterior fornix. On digital examination, it was found to be a hollow cylindrical body around $3.5 \mathrm{~cm}$ diameters with grooves on inner aspect, deeply impacted and fixed in left inferior-lateral aspect of cervix. Bimanual examination revealed an atrophic uterus and no adenexal mass.

She denied any history of any accidental or intentional insertion of any foreign body in vagina. The patient was admitted and put on parenteral antibiotics. A radiograph and ultrasonography (USG) of pelvis was done which confirmed the clinical examination findings (Fig. 1). After 48 hours of antibiotic cover, patient was taken up for examination under anaesthesia and removal of foreign body. On examination under anaesthesia (EUA), it was found that the foreign body was deeply impacted. Tissue dissection was done along the margins of the foreign body and it was held with four Zeppelin clamps. Using rotating clockwise-anticlockwise movements, the foreign body was taken out and removed from vagina (Figs. 2, 3). The base of the tunnel was explored for any intra peritoneal connection and liberal vaginal irrigation with povidoneiodine was done. It was a plastic foreign body with an aluminum rim, hexagonal in shape. Postoperative period was uneventful. The patient was continued on antibiotics for five days postoperatively and was discharged on sixth postoperative day.

\section{Discussion}

Some objects like tampons, vaginal suppositories, medications and contraceptive devices are designed for use in a woman's vagina. Others are not intended to be inserted and may be placed there accidentally or intentionally. These are referred to as "foreign bodies". These foreign bodies may produce symptoms or be asymptomatic for long periods of time. Small objects inserted into the vagina do not generally cause pain. Unusual objects which are larger than the customary vaginal diameter or size of the introitus may cause distension pain. Other objects may cause pain due to sharp and irregular edges and because of associated infections, which might superimpose.

Long standing presence of a foreign body in vagina may cause vesicovaginal fistula and urinary incontinence $[1,2]$. It may be complicated by vaginal stenosis or near complete obstruction [3]. Metallic objects, except aluminum, are radio-opaque. Most animal bones and all glass bodies are opaque. Most plastic and wooden bodies and most fish bones may not be opaque [4].

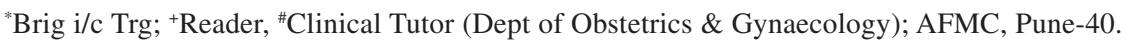

Received : 04.10.09; Accepted : 14.05.10_E-mail : choprasanjiv@ rediffmail.com 


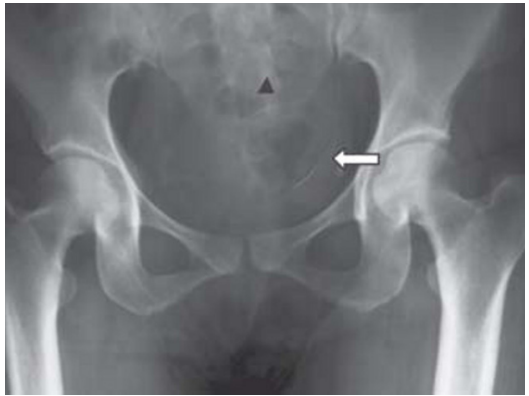

Fig. 1 : Radiograph of pelvis showing foreign body in situ (marked with an arrow).

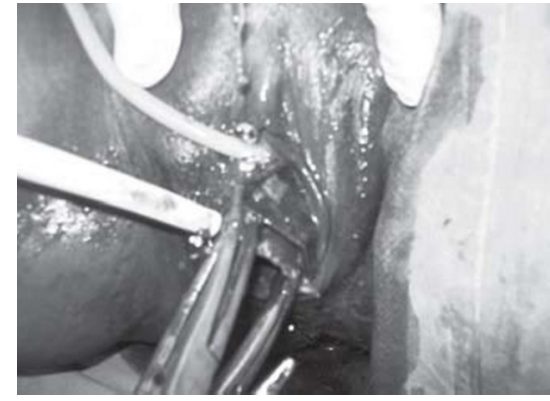

Fig. 2 : Foreign body being taken out of vagina, held with clamps.

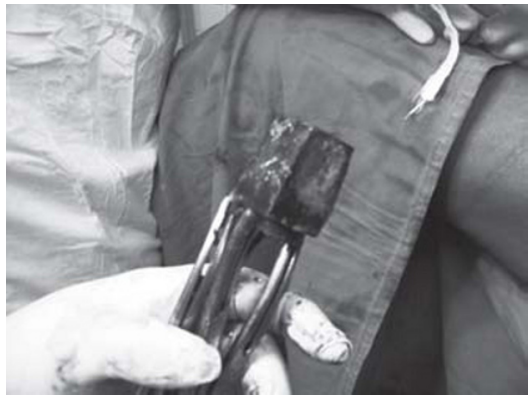

Fig. 3 : Foreign body taken out of vagina, still held with clamps.
Vaginal foreign bodies are very commonly seen in children. Adolescent girls may present with foreign bodies, which primarily consist of forgotten tampons or broken portions of condoms. Adults may also present to the emergency department with vaginal foreign bodies which may have been placed there intentionally as part of a sexual encounter or placed as part of an episode of abuse or to induce abortion illegally.

While a variety of symptoms may result from a foreign body in the vagina, the most common symptoms are bleeding or foulsmelling vaginal discharge [5]. Less common symptoms may include pain or urinary discomfort. Sometimes, though rarely, foreign bodies produce a systemic infection in circumstances such as severe immunocompromise or disruption of the vaginal wall with secondary infection. Perforation through the vagina into the abdominal cavity may also result in acute abdominal symptoms.

The presence of a foreign body may alter the normal vaginal flora, thus resulting in failed repeated efforts to treat a case presumed to be vaginitis. Repeated use of antibiotics or other medications will not ameliorate the symptoms if a foreign body persists. Foreign bodies left in the vagina very rarely lead to serious complications. However, the medical literature is replete with several case reports of pelvic abscess and subsequent scarring.

Children may be unable to provide history of an object placed in the vagina; however, some may come forward with history of losing an object in their vagina. In addition to obtaining specific information about a possible vaginal foreign body, a thorough history, general and systemic physical examination are warranted. It is also appropriate at this time to ask questions related to sexual activity and sexual or physical abuse. The possibility of sexual abuse especially in girls with a vaginal foreign body, should always be explored [6].

An adolescent patient may easily have a foreign body removed from the vagina in the OPD setting. This may also hold true for adults. Proper visualization of the foreign body using a speculum and removal with a forceps may be the most efficient treatment. Unusual objects or those that may disrupt the vaginal wall may require anaesthesia for removal and to complete a thorough examination of the vagina and cervix. If an object has been present in the vagina for a long time, it may cause erosion into the wall of the vagina. Acute placement of an unusual object in the vagina may cause perforation of the vaginal wall and secondary symptoms of an intra-abdominal infection.

Although examination generally reveals the presence of a foreign body, some imaging techniques may also be helpful. Methods to rule out a vaginal foreign body include a careful history taking and genital examination, bimanual rectoabdominal palpation, pelvic USG, plain pelvic radiography, vaginography and magnetic resonance imaging (MRI). MRI is supposed to be the best technique for evaluating vaginal foreign bodies [7]. The use of MRI has increased the localization of nonmetallic objects missed by USG and radiologic studies [8, 9]. Procedures such as vaginoscopy and continuous flow vaginoscopy with a $4 \mathrm{~mm}$ hysteroscope under general anaesthesia and/or vaginal irrigation with normal saline are very useful for detection and management of a vaginal foreign body [8, 9]. Vaginoscopy is indicated for recurrent vulvovaginitis unresponsive to improved local hygiene and medical therapy or associated with bleeding and for suspicion of a foreign body, neoplasm or congenital anomaly.

The ideal management of an uncomplicated foreign body is its removal. This is generally the only treatment necessary. In cases, where there has been invasion into contiguous anatomies, specific repair procedures may be indicated.

\section{Conflicts of Interest}

None identified

\section{References}

1. Biswas A, Das HS. An unusual foreign body in the vagina producing vesicovaginal fistula. Indian Med Assoc 2002; 100: 257-9.

2. Picurelli L, Lopez Olmos J, Sendra A. Vesicovaginal fistula 
caused by foreign body in the vagina. Actas Urol Esp 1997; 21: 511-2.

3. Simon DA, Berry S, Brannian J, Hanson K. Recurrent, purulent vaginal discharge with long standing presence of a foreign body and vaginal stenosis. J Paediatric Adolosc Gynecol 2003; 16: 361-3.

4. Hunter TB, Taljanovic MS. Foreign bodies. Radio Graphics 2003; 23: 731-57.

5. Dahiya P, Sangwan K, Khosla A. Foreign body in vagina: an uncommon cause of vaginitis in children. Indian J Paediatr 1999; 66: 466-7.
6. Herman - Giddens ME. Vaginal foreign bodies and child sexual abuse. Arch Paediatric Adolesc Med 1994; 148: 195-200.

7. Kihara M, Sato N, Kimura H. Magnetic resonance imaging in the evaluation of vaginal foreign bodies in a young girl. Arch Gynecol Obstet 2001; 265: 221-2.

8. Smith Y, Berman DR, Quint EH. Premenarchal vaginal discharge: finding of procedures to rule out foreign bodies. J Paediatr Adolesc Gynecol 2002; 15: 227-30.

9. Golan A, Lurie S, Sagiv R, Glezerman M. Continuous flow vaginoscopy in children and adolescents. J Am Assoc Gynecol Laparosc 2000; 7: 526-8.

\section{Quiz}

\section{Radiological Quiz}

\section{Lt Col U Rajesh*}

MJAFI 2010; $66: 268$

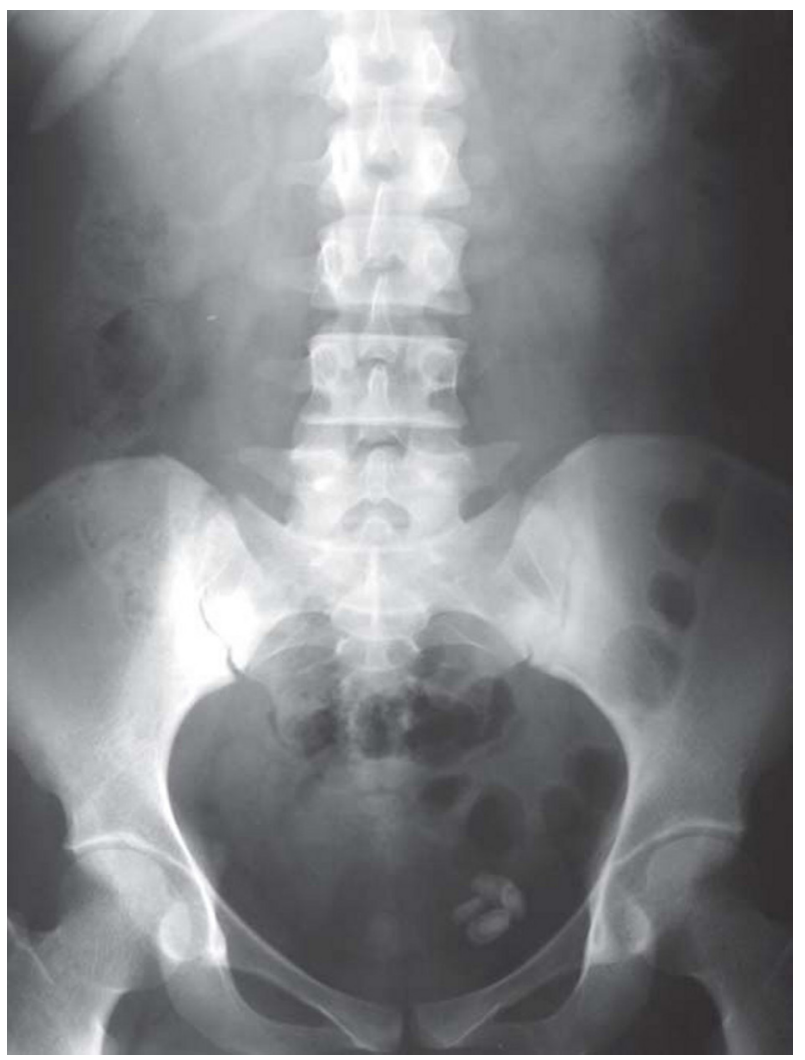

Fig. 1: Radiograph of the KUB region shows multiple calculi in the left hemipelvis, the largest measuring $18 \mathrm{~mm}$ x $10 \mathrm{~mm}$.

Answer to Radiological Quiz pg 278.
Thirty one year old female presented with complaints of recurrent pain in left side of abdomen. Pain was colicky, intermittent and used to radiate to the left groin. Patient also had dysuria and urgency. Physical examination was within normal limits. Urine analysis and culture revealed symptomatic bacteriuria suggestive of urinary tract infection. Her renal function tests were within normal limits.

Fig. 1 shows radiograph of the kidney, ureter and bladder (KUB) region. Patient underwent ultrasonography (USG) of KUB region. Fig. 2 shows transverse sonogram of the bladder.

What is your diagnosis?

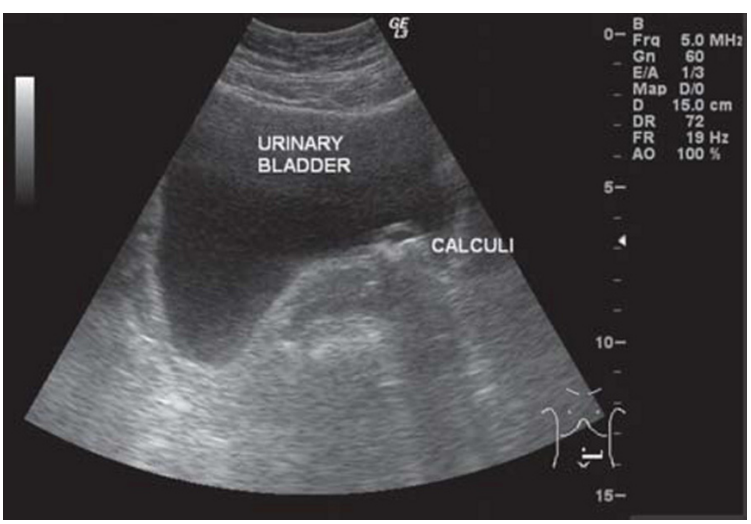

Fig. 2: Transverse sonogram of the bladder shows multiple echogenic foci, casting distal acoustic shadowing, in the region of left uretero-vesical junction.

*Graded Specialist (Radiodiagnosis), 150 General Hospital, C/o 56 APO, PIN - 900129

Received : 30.09.09; Accepted : 10.01.10

E-mail : uddandamrajesh@yahoo.com 